\title{
QPOS OF BY CAM IN LOW AND HIGH STATES
}

\author{
E. P. PAVLENKO ${ }^{1}$, S. YU. SHUGAROV ${ }^{2}$, S. V. ANTIPIN ${ }^{2}$, \\ D. A. SOKOLOV ${ }^{2}$, G. BORISOV ${ }^{2}$, I. BUDZINOVSKAYA ${ }^{1}$ \\ 1. Crimean Astrophysical Observatory, Crimea 334413, \\ Ukraine. e-mail: pavlenko@crao.crimea.ua \\ 2. Sternberg Astronomical Institute, Universitetsky avenue, 13, \\ MoscowV-234, 119899, Russia.e-mail: shugarov@sai.msu.su
}

\begin{abstract}
We present the analysis of $B V$ photometry of BY Cam based on observations carried out in Crimea. The long QPOs in brightness were detected in both high and low brightness states. Their typical periods, equal to 6, 12 and $24 \mathrm{~m}$, are found. The amplitude of the QPOs depends on the phase of $P_{\text {beat }}$.
\end{abstract}

\section{Results}

BY Cam is an AM Her type binary discoveréd by Remillard et al. (1986). It is the second, after the polar V1500 Cyg, to show asynchronism of the white dwarf's spin with respect to the binary and a fast synchronization of the components (Mason et al. 1995). BY Cam shows very complicated behaviour in optical light due to the existence of several periods: orbital, orbital side--band, spin and spin-orbit beat periods (Mason et al. 1995).

The observations of BY Cam were carried out at the Crimean Astrophysical Observatory and Sternberg Astronomical Institute with the 0.5, 0.6 and $1.25 \mathrm{~m}$ telescopes using the $B V R$ system during 25 nights from 1989 up to 1994 . The long-term $B V R$ light curve is shown by Sokolov, Shugarov \& Pavlenko (1996).

One of the most intriguing and unresolved problems in studying BY Cam is the evidence of the so-called long (tens of minutes) quasi-periodic brightness oscillations. They appear both in a low and high brightness state and have a varying amplitude, which sometimes increases up to $0.3 \mathrm{mag}$.

Adopting $P_{\text {beat }}=14.11 \mathrm{~d}$ and $P_{\text {spin }}=0.1384238 \mathrm{~d}$ (Mason et al. 1995), we have analysed the behaviour of the QPOs amplitude with the phase of $P_{\text {beat }}$ (or with $O-C$ of $P_{\text {spin }}$ for both minima and maxima). The elements: 

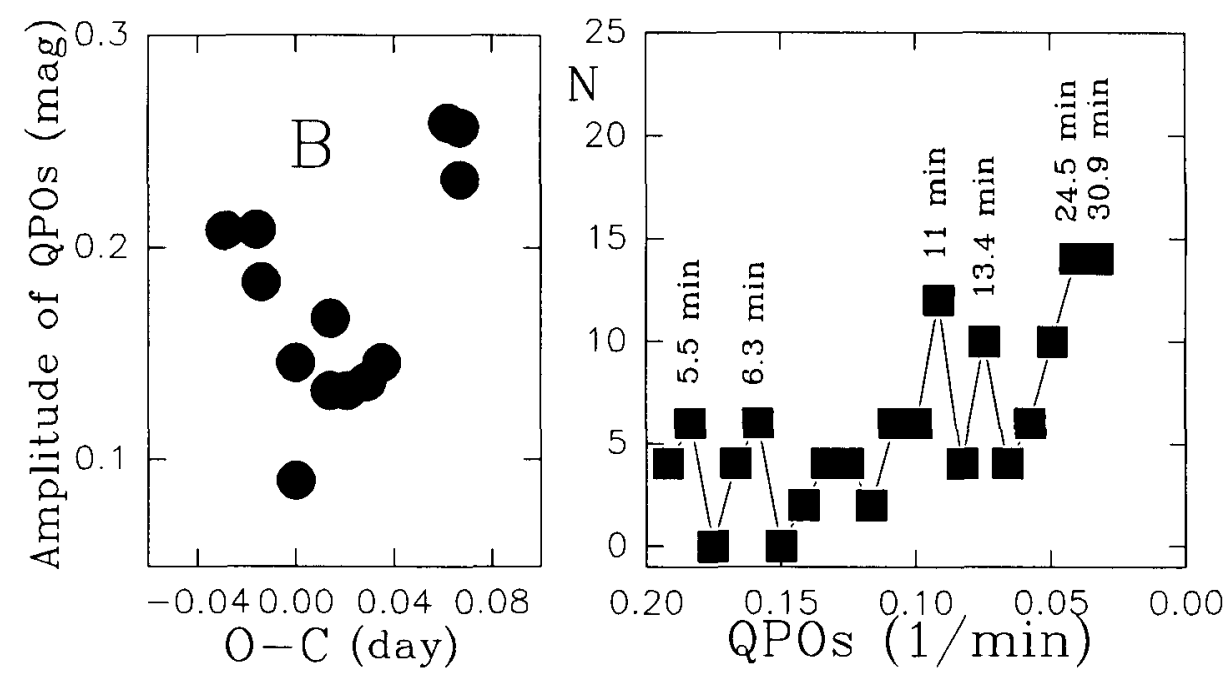

Figure 1. Left: Dependence of QPO amplitude on the $O-C$ of $P_{\text {spin }}$. Right: The histogram of the most significant QPOs detected for all observations in $V$.

$C_{\max }=2447540.288+0.1384238 E, C_{\min }=2447591.443+0.1384238 E$ were used. We have seen a strong dependence in $B$ (Fig. 1 left) but not in $V$.

Over five years we have found several stable, (multiple?) QPOs (Fig. 1 right), which are close to 6,12 and $24 \mathrm{~m}$. Note that the asynchronous magnetic nova V1500 Cyg also exhibited QPOs with typical time-scales of $17 . .26 \mathrm{~m}$ (Pavlenko 1992). The analysis shows that these QPOs do not have a stable zero phase, producing a broad peak on a periodogram. But the intermediate brightness state exhibited a stable $5.8 \mathrm{~m}$ QPO over $100 \mathrm{~d}$. Acknowledgments. This research was supported by a grant from the Center for Cosmoparticle Physics 'Cosmion'. We also are grateful to the Keele organising committee for the possibility of attending this meeting.

\section{References}

Mason, P.A., Chanmugam, G., Andronov, I.L., et al., 1995, in "Cataclysmic Variables", eds A. Bianchini et al., Kluwer, p426

Pavlenko, E.P., 1992, Izv. Krym. Astroph. Obs., 86, 55

Remillard, R.A., Bradt, H.V., McClintock, J.E., et al., 1986, Ap. J., 302, L11

Sokolov, D.A., Shugarov S.Yu., Pavlenko E.P., 1996, these proceedings, p219 